\title{
Therapeutic Effect of 0.1\% Topical Tacrolimus for Childhood Interstitial Keratitis Refractory to Cyclosporine
}

\author{
Takeshi Joko ${ }^{1}$, Atsushi Shiraishi ${ }^{1}$, Miki Ogata $^{2}$ and Yuichi Ohashi ${ }^{1}$ \\ ${ }^{1}$ Department of Ophthalmology, Ehime University Graduate School of Medicine \\ ${ }^{2}$ Department of Ophthalmology, Matsuyama Shimin Hospital
}

\begin{abstract}
Purpose: To report our findings in a case of childhood refractory interstitial keratitis successfully treated with $0.1 \%$ topical tacrolimus.

Methods: A 12-year-old boy presented with a 3-year history of interstitial keratitis. For the recurrent interstitial keratitis he had been treated with topical and systemic acyclovir, steroids, and topical cyclosporine for 3 years. Our examinations revealed severe stromal infiltrates and neovascularization. Treatment was changed from topical $0.5 \%$ cyclosporine to topical $0.1 \%$ tacrolimus combined with topical acyclovir and betamethasone.

Results: After 2 weeks of treatment with topical tacrolimus, the degree of stromal infiltrates decreased. Although the improvements were slow, the stromal infiltrates resolved somewhat, and neovascularization and topical acyclovir and betamethasone were tapered and stopped in 18 months. Since then, the patient has not shown any recurrence for 9 months without medication.

Conclusion: Our findings indicate that topical tacrolimus should be considered for treating refractory interstitial keratitis in children. (J Nippon Med Sch 2016; 83: 31-34)
\end{abstract}

Key words: childhood, refractory interstitial keratitis, tacrolimus

\section{Introduction}

Interstitial keratitis, also known as immune stromal keratitis, is an inflammation of the corneal stroma without primary involvement of the corneal epithelium or endothelium. The pathogenic mechanism of interstitial keratitis is thought to be an antigen-antibodycomplement-mediated immune disease characterized by nummular opacities, stromal infiltrations, and immune rings. The chronicity of these reactions can lead to hypersensitive immune disease characterized by disciform edema and neovascularization of the stroma.

Before the introduction of antibiotics, syphilis was recognized as the most common cause of interstitial keratitis; however, recent reports indicate that herpes simplex virus (HSV) is now the leading cause of interstitial keratitis and is followed by idiopathic interstitial keratitis, syphilis, and varicella-zoster virus $(\mathrm{VZV})^{1}$. The principle management of interstitial keratitis is with specific drugs against the causative agent combined with im- mune suppressants, such as corticosteroids and cyclosporine $e^{2-4}$.

We report a case of childhood interstitial keratitis that was successfully treated with topical $0.1 \%$ tacrolimus. The interstitial keratitis was initially suspected to be zoster sine herpete that was refractory to corticosteroids and cyclosporine.

\section{Case Report}

The patient was a 12-year-old boy with recurrent interstitial keratitis who had been treated for 3 years with topical and systemic acyclovir, corticosteroids, and topical cyclosporine. He was referred to the Ehime University Hospital on September 14, 2007, at 9 years of age for uveitis of unknown cause and had been treated with topical $0.5 \%$ levofloxacin 3 times a day.

The protocol for the treatment of this case was approved by the institutional review board of Ehime University No. 1312004, and informed consent for the exami-

Correspondence to Atsushi Shiraishi, Department of Ophthalmology, Ehime University Graduate School of Medicine, Shitsukawa, Toon, Ehime 791-0295, Japan

E-mail: shiraia@m.ehime-u.ac.jp

Journal Website (http://www.nms.ac.jp/jnms/) 


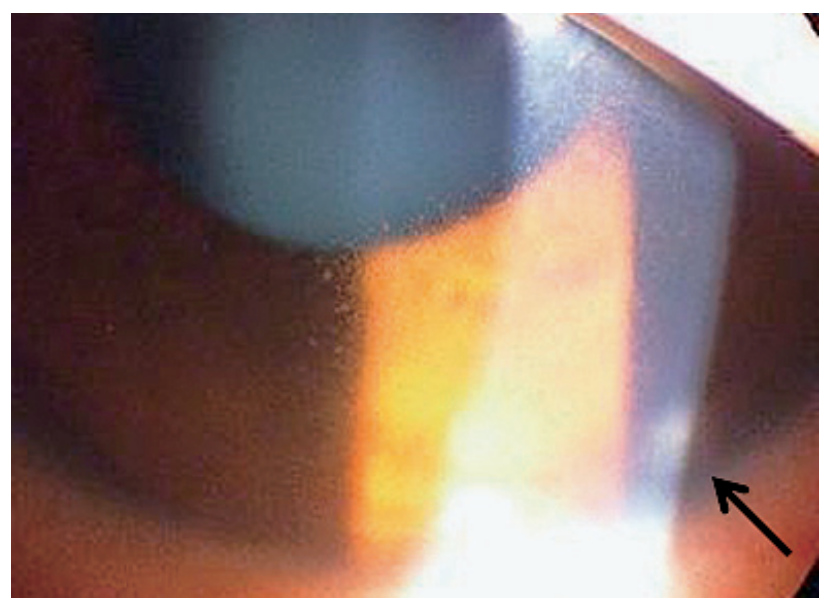

Fig. 1 Slit-lamp photographs of the cornea of a 9-yearold boy at the initial visit on September 14, 2007. Slit-lamp examination of the left eye shows mild mutton fat keratoprecipitates, mild corneal stromal infiltration at $4 \mathrm{o}^{\prime}$ clock (arrow), and mild injection.

nation and treatments was obtained from the patient's parents. The procedures used conformed to the tenets of the Declaration of Helsinki.

At the initial examination on September 14, 2007, the patient's best-corrected visual acuity was 24/20 right eye (OD) and 24/20 left eye (OS), and the intraocular pressure was $14 \mathrm{~mm} \mathrm{Hg}$ OD and $11 \mathrm{~mm} \mathrm{Hg}$ OS. Slit-lamp examination of the left eye showed mild mutton fat keratoprecipitates with inflammatory cells in the anterior chamber, mild corneal stromal infiltration at 4 o'clock, and mild injections (Fig. 1). The laboratory data did not indicate what the cause of the uveitis might be.

The patient was treated with systemic prednisolone (10 mg/day) and topical $0.1 \%$ betamethasone added to levofloxacin for the uveitis. After this treatment was started, the keratoprecipitates and corneal stromal infiltration improved, and diffuse scleritis developed with the continuous treatment. Because the cause of the uveitis was unknown, screening laboratory examinations were performed for uveitis, including associated systemic diseases, infections, and infestations. However, the laboratory examinations did not indicate what had caused the uveitis. On December 27, 2007, the patient showed nummular corneal stromal infiltrations (Fig. 2).

Although the serological examination was negative for HSV and positive for only VZV immunoglobulin G (X 6.6), we suspected that the sclerokeratitis might have been caused by a herpetic infection because of the poor response to prednisolone and the clinical observations. Because of the clinical observations for therapeutic diag-

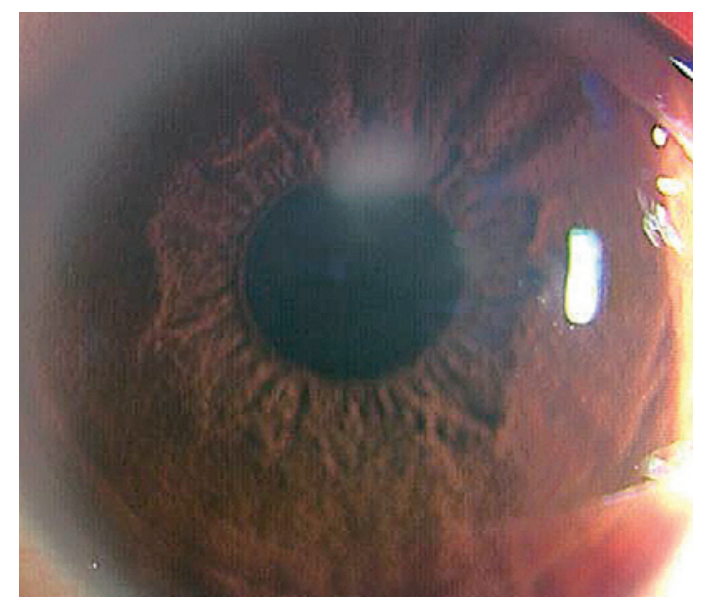

Fig. 2 Slit-lamp photographs 3 months later on December 27, 2007. Slit-lamp examination of the left eye showed nummular corneal stromal infiltrations.

nosis, systemic acyclovir ( $80 \mathrm{mg} / \mathrm{kg} /$ day) and 3\% acyclovir ointment (5 times a day) were added to the systemic prednisolone $(10 \mathrm{mg} /$ day) and topical $0.1 \%$ betamethasone. After treatment with this regimen, the scleritis improved within 1 week and the corneal stromal infiltration improved over the following months. Therefore, the systemic acyclovir and prednisolone were tapered and stopped 1 month later. The $0.1 \%$ betamethasone was replaced with $0.1 \%$ fluorometholone, and then the topical acyclovir and fluorometholone were stopped 7 months later. Thereafter, the patient visited our outpatient clinic without any recurrence.

When the patient was examined on March 5, 2010, corneal injections and nummular stromal infiltration with neovascularization were detected (Fig. 3). He was suspected to have recurrent herpetic zoster interstitial keratitis, and treated was started with systemic acyclovir (80 $\mathrm{mg} / \mathrm{kg} /$ day), systemic prednisolone (10 mg/day), topical acyclovir ointment (5 times a day), and $0.1 \%$ topical fluorometholone (4 times a day). The nummular opacities improved, and the systemic acyclovir and prednisolone were tapered and stopped after 1 month. However, on April 9, 2010, neovascularization and interstitial keratitis recurred, and the topical $0.1 \%$ fluorometholone (4 times a day) was replaced with topical $0.1 \%$ betamethasone (6 times a day), and systemic prednisolone (10 mg/day) was added to the topical acyclovir ointment (5 times a day). No improvement was observed, and, therefore, on June 22, 2010, topical $0.5 \%$ cyclosporine (5 times a day) was added to topical $0.1 \%$ betamethasone (6 times a day), acyclovir ointment (5 times a day), and systemic prednisolone (10 mg/day). However, even after such an 


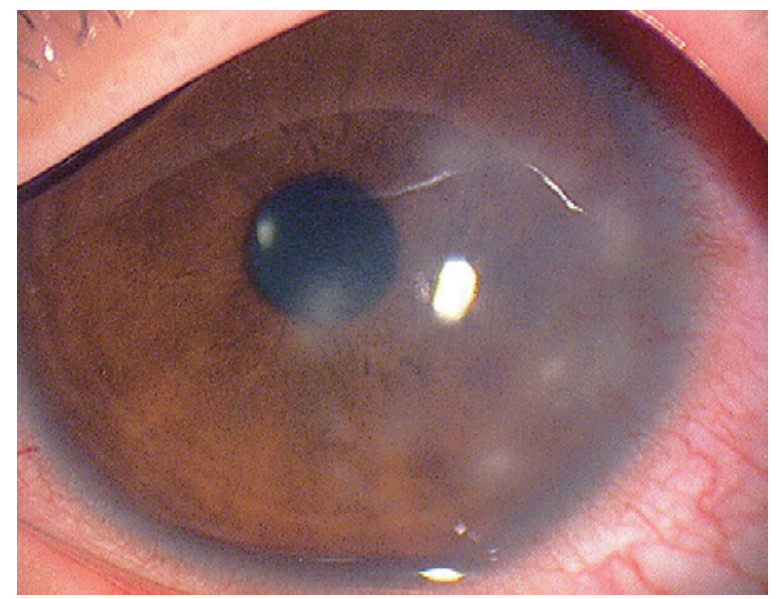

Fig. 3 Slit-lamp photographs 2 years 6 months after the initial examination on March 05, 2010. Slit-lamp examination of the left eye shows injection and nummular corneal stromal infiltration with neovascularization.

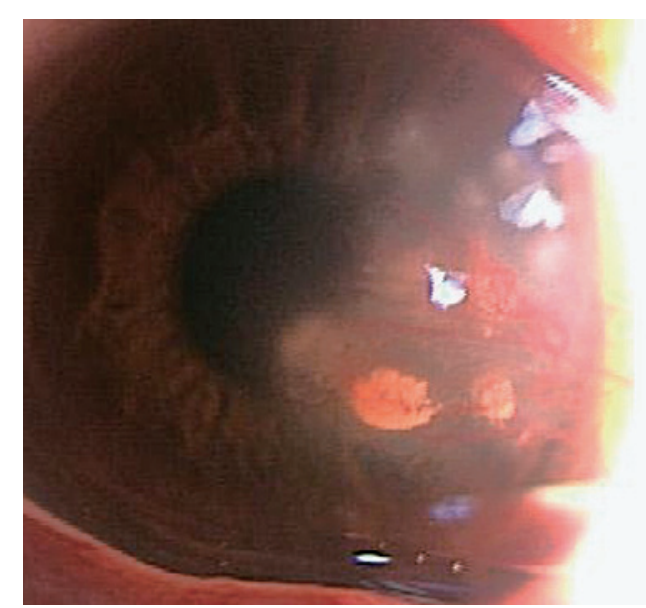

Fig. 4 Slit-lamp photographs 3 years 3 months after the initial examination on December 28, 2010. Slitlamp examination of the left eye shows stromal infiltration and neovascularization.

intensive therapy, neovascularization and interstitial keratitis continued to recur (Fig. 4).

Therefore, on December 28, 2010, the $0.5 \%$ topical cyclosporine (5 times a day) was replaced with topical $0.1 \%$ tacrolimus (2 times a day). Two weeks after the start of treatment with topical $0.1 \%$ tacrolimus, the neovascularization and interstitial keratitis improved and systemic steroid was tapered and stopped. Three months later, the interstitial keratitis had almost completely resolved and topical acyclovir and betamethasone began to be tapered (Fig. 5). Since then, the patient has been treated with topical $0.1 \%$ tacrolimus (2 times a day) and topical $0.1 \%$ betamethasone (2 times a day) and tapered and stopped

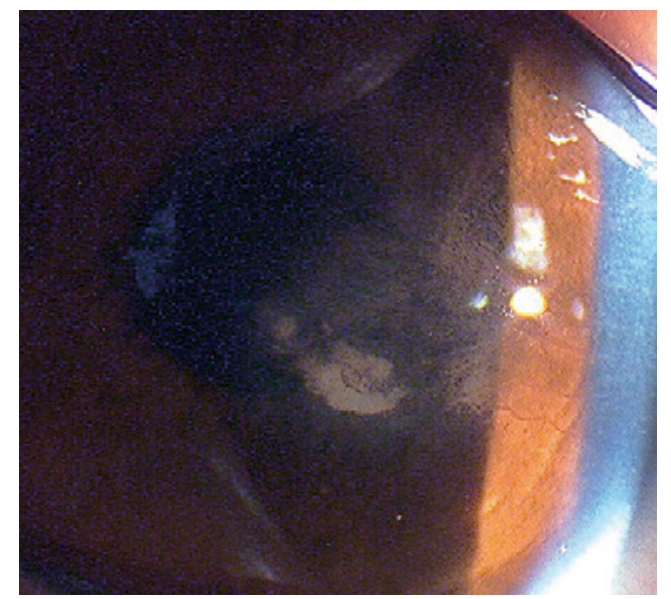

Fig. 5 Slit-lamp photographs 3 month after $0.1 \%$ tacrolimus treatment. Slit-lamp examination of the left eye showed resolution of the stromal infiltration and decreased neovascularization.

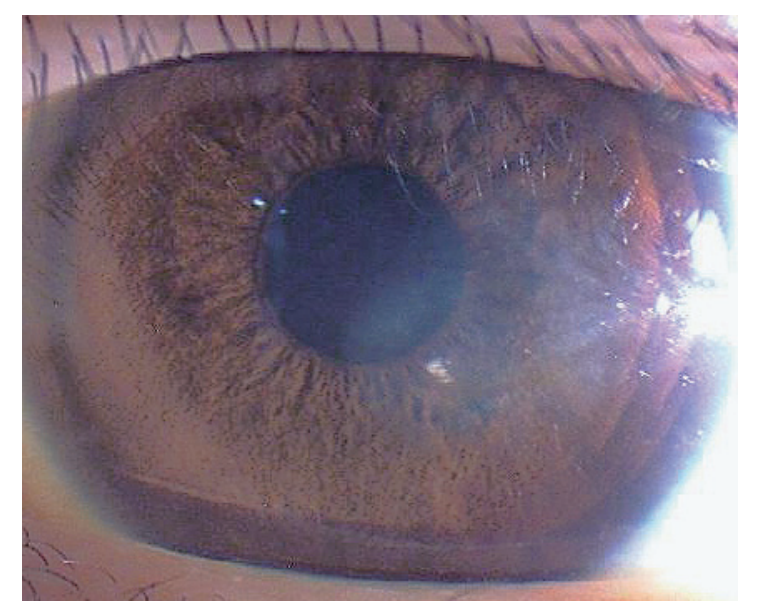

Fig. 6 Slit-lamp photographs 9 month after cessation of $0.1 \%$ tacrolimus treatment.

Slit-lamp examination of the left eye showed resolution of the stromal infiltration and scarred vascularization.

in 18 months. Since then, he has not shown any recurrence for 9 months, the best-corrected visual acuity improved to $24 / 20 \mathrm{OD}$ and $24 / 20 \mathrm{OS}$, and the intraocular pressure was $10 \mathrm{~mm} \mathrm{Hg} \mathrm{OD}$ and $11 \mathrm{~mm} \mathrm{Hg}$ OS on January 22, 2013 (Fig. 6).

\section{Discussion}

Interstitial keratitis is a chronic corneal inflammation that can lead to severe corneal scarring and visual decrease. At present, the most common cause of interstitial keratitis is HSV, and HSV-interstitial keratitis is treated with antiviral drugs and immune suppressants, such as corticosteroids ${ }^{1}$. Although herpes zoster ophthalmicus in 
childhood and zoster sine herpete ophthalmicus in adults are rare, to our knowledge sine herpete ophthalmicus has not been reported in children. However, zoster sine herpete has been reported in children with Ramsay Hunt syndrome ${ }^{5}$.

In the present case, a definitive diagnosis could not be made because there was no unambiguous serological data, but interstitial keratitis caused by zoster sine herpete may be suspected by the typical clinical course. The effectiveness of cyclosporine A against refractory interstitial keratitis due to HSV has been demonstrated ${ }^{4}$; however, topical cyclosporine had no effect in the present case.

Tacrolimus is a potent immunosuppressive macrolide, and its mechanism of action is similar to that of cyclosporine. However, the potency of tacrolimus is 30 times greater than that of cyclosporine ${ }^{6}$. Its clinical efficacy has been demonstrated against refractory ocular surface inflammatory diseases ${ }^{7-9}$. Therefore, we replaced topical cyclosporine with topical tacrolimus, which was extremely effective against the uncontrolled deep stromal keratitis. The effectiveness of tacrolimus supports our hypothesis that our case was a herpes infection because tacrolimus is known to inhibit the transcription of interleukin 2, which inhibits the proliferation of $\mathrm{T}$ lymphocytes $^{10}$. Although the exact mechanism of how VZV causes interstitial keratitis is not known, it has been suggested that interleukin 2-mediated $\mathrm{T}$ lymphocytes play an important role in interstitial keratitis due to $\mathrm{HSV}^{2,11,12}$.

In conclusion, our case of childhood refractory interstitial keratitis was most likely due to zoster sine herpete and was successfully treated with $0.1 \%$ topical tacrolimus. Thus, topical tacrolimus should be considered in treating cases of childhood refractory interstitial keratitis.

Conflict of Interest: The authors declare no conflict of inter- est.

\section{References}

1. Schwartz GS, Harrison AR, Holland EJ: Etiology of immune stromal (interstitial) keratitis. Cornea 1998; 17: 278281.

2. Heiligenhaus A, Steuhl KP: Treatment of HSV-1 stromal keratitis with topical cyclosporin A: a pilot study. Graefes Arch Clin Exp Ophthalmol 1999; 237: 435-438.

3. McGill J: The enigma of herpes stromal disease. Br J Ophthalmol 1987; 71: 118-125.

4. Rao SN: Treatment of herpes simplex virus stromal keratitis unresponsive to topical prednisolone $1 \%$ with topical cyclosporine $0.05 \%$. Am J Ophthalmol 2006; 141: 771-772.

5. Ogita S, Terada K, Niizuma T, Kosaka Y, Kataoka N: Characteristics of facial nerve palsy during childhood in Japan: frequency of varicella-zoster virus association. Pediatr Int 2006; 48: 245-249.

6. Almawi WY, Melemedjian OK: Clinical and mechanistic differences between FK506 (tacrolimus) and cyclosporin A. Nephrol Dial Transplant 2000; 15: 1916-1918.

7. Joseph MA, Kaufman HE, Insler M: Topical tacrolimus ointment for treatment of refractory anterior segment inflammatory disorders. Cornea 2005; 24: 417-220.

8. Kymionis GD, Kankariya VP, Kontadakis GA: Tacrolimus ointment $0.03 \%$ for treatment of refractory childhood phlyctenular keratoconjunctivitis. Cornea 2012; 31: 950952.

9. Miyazaki D, Tominaga T, Kakimaru-Hasegawa A, Nagata Y, Hasegawa J, Inoue Y: Therapeutic effects of tacrolimus ointment for refractory ocular surface inflammatory diseases. Ophthalmology 2008; 115: 988-992 e5.

10. Martinez-Martinez S, Redondo JM: Inhibitors of the calcineurin/NFAT pathway. Curr Med Chem 2004; 11: 9971007.

11. Gangappa S, Deshpande SP, Rouse BT: Bystander activation of CD4(+) $\mathrm{T}$ cells can represent an exclusive means of immunopathology in a virus infection. Eur J Immunol 1999; 29: 3674-3682.

12. Heiligenhaus A, Bauer D, Zheng M, Mrzyk S, Steuhl KP: CD4+ T-cell type 1 and type 2 cytokines in the HSV-1 infected cornea. Graefes Arch Clin Exp Ophthalmol 1999; 237: 399-406.

(Received, June 16, 2015)

(Accepted, July 29, 2015) 